\title{
ANÁLISE DE CRESCIMENTO DO AMENDOINZEIRO (Arachis hypogaea L.) COM RELAÇÃO $\bar{A}$ INFESTAÇÃO DE PRAGAS
}

\author{
Paulo R. C. Castro **
}

\section{RESUMO}

\begin{abstract}
Em condições de campo, estudou-se através da análise de crescimento, os efeitos de pragas do amendoinzeiro nos fatores fisiologicos ligados à capacidade de produção da cultura. Verificou-se que em um mesmo sistema eculógico, o tratamento da cultura com defensivo incrementa o indice de área foliar e a relação de área foliar, devido a manutenção da população de pragas em nivel inferior, com relação ao cultivo sem aplicação do inseticida. A taxa de assimilação aparente e a taxa de crescimento relativo revelaram-se superiores no tratamento sem defensivo, indicando que o ganho em produtos de fotossíntese e a produção de matéria seca por unidade foliar, são mais elevados neste tratamento.
\end{abstract}

\section{INTRODUÇAO}

A análise de crescimento das culturas tem sido muito utilizada para a obtenção de parâmetros fisiológicos indicativos de métodos seguros para o aumento da produtividade.

Desde os estudos fisiológicos comparativos do desenvolvimento de culturas (WATSON, 1947), até a verificação do efeito da densidade de plantio no aproveitamento da energia luminosa (ALVIM \& ALVIM, 1969), uma série de importantes trabalhos têm sido realizados (MONSELISE, 1953; HUERTA \& ALVIM, 1962; VINOGRADOV, 1968; MONTOJOS \& MAGALHAES, 1971; BRANDES et al., 1973).

Diversas espécies de cigarrinhas causam danos foliares à macieira, e essas injúrias são acompanhadas por uma redução na fotossíntese aparente; sendo que as distintas espécies do inseto, em igual número, afetam com diferente intensidade o metabolismo foliar. A fotossíntese aparente

* Entregue para publicação em 29/08/1974.

** Departamento de Botânica. E. S. A. «Luiz de Queiroz» - U. S. P. 
nas folhas de macieira pode ser reduzida no início da estação de crescimento, quando a população de cigarrinha é moderadamente baixa, e assim a capacidade fotossintética das folhas injuriadas é debilitada de forma permanente, sendo que o controle preventivo desses insetos torna-se, desta forma, importante (MARSCHALL et al., 1942).

Para verificar o efeito de pragas do amendoinzeiro (Arachis hypogaea L.) no desenvolvimento da cultura, determinaram-se através da análise de crescimento das plantas, os fatores fisiológicos considerados mais intimamente ligados à capacidade de produção do vegetal, tais como, o índice de área foliar, a relação de área foliar, taxa de assimilação aparente e a taxa de crescimento relativo (GRANGIER JR. \& ALVIM, 1964; CASTRO et al., 1972).

\section{MATERIAL E MÉTODOS}

O trabalho foi realizado na região de Jaboticabal, onde o amendoinzeiro é extensamente cultivado. O plantio foi efetuado em um solo tipo Latosolo Roxo, em 9/3/1971, «cultura da seca», utilizando-se o amendoinzeiro Arachis hypogaea L. cv. 'Tatú-53'.

0 ensaio foi composto de dois tratamentos com dez repetições, sendo cada parcela constituida por duas linhas de dez metros de comprimento. 0 espaçamento foi de $60 \times 10 \mathrm{~cm}$ e ambos os tratamentos encontravam-se sob uma mesma condição ecológica, onde tínhamos, um tratamento sem aplicação de defensivo e outro tratamento onde ef etuaram-se aplicações semanais de Paratiom metílico a $0,09 \%$, do concentrado emulsionável a $60 \%$.

Verificou-se que as principais pragas que ocorreram durante o ciclo do amendoinzeiro foram, pela ordem decrescente de importância, o tripes Enneothrips flavens Moulton, 1941, a vaquinha Diabrotica speciosa (Germar, 1824) e a lagarta do pescoço vermelho Stegasła bosquella (Chambers, 1875).

Fizeram-se coletas de 150 plantas em cada 14 dias, no decorrer do ciclo da cultura, para cada tratamento. Em seguida essas plantas foram desfolhadas, procedeu-se à secagem das folhas em estufa e suas áreas foram determinadas através do conhecimento do peso seco de áreas conhecidas de amostras das mesmas. Também procedeu-se à secagem do restante das plantas. Foram realizadas 10 colheitas de plantas. Calculou-se, o índice de área foliar (IAF), a relação de área foliar (RAF), a taxa de assimilação aparente (TAA) e a taxa de crescimento relativo (TCR), para os dois tratamentos, no decorrer do ciclo da cultura, através das fórmulas seguintes (BLACKMAN \& WILSON, 1951):

$$
\mathrm{IAF}=\frac{\mathrm{A}}{\mathrm{S}}
$$




$$
\begin{aligned}
& \mathrm{RAF}=\frac{\mathrm{A}}{\mathrm{P}}=\mathrm{dm}^{2} / \mathrm{g} \\
& \mathrm{TAA}=\frac{\left(\mathrm{P}_{2}-\mathrm{P}_{1}\right)}{\left(\mathrm{A}_{2}-\mathrm{A}_{1}\right)} \cdot \frac{\left(\log _{e} \mathrm{~A}_{2}-\log _{e} \mathrm{~A}_{1}\right)}{\left(\mathrm{T}_{2}-\mathrm{T}_{1}\right)}=\mathrm{g} / \mathrm{dm}^{2} / \mathrm{dia} \\
& \mathrm{TCR}=\frac{\left(\log _{\mathrm{e}} \mathrm{P}_{2}-\operatorname{LogeP}_{1}\right)}{\left(\mathrm{T}_{2}-\mathrm{T}_{1}\right)}=\mathrm{g} / \mathrm{g} / \mathrm{dia}
\end{aligned}
$$

$\mathrm{A} / \mathrm{S}=$ razão, área foliar/área da superfície do terreno em uma mesma colheita

$\mathrm{A} / \mathrm{P}=$ razão, área foliar/peso seco de uma mesma colheita, em $\mathrm{dm}^{2} / \mathrm{g}$ $\mathrm{P}_{2}-\mathrm{P}_{1}=$ diferença de peso seco em $\mathrm{g}$, entre duas amostras consecutivas $\mathrm{A}_{2}-\mathrm{A}_{1}=$ diferença de área foliar, em $\mathrm{dm}^{2}$, entre as mesmas amostras $\mathrm{T}_{2}-\mathrm{T}_{1}=$ tempo transcorrido em dias, entre as colheitas

\section{RESULTADOS E DISCUSSÁO}

As plantas tratadas com o inseticida apresentaram sempre índices de área foliar superiores àquelas não tratadas; isto revela que naquele mesmo sistema ecológico os insetos causaram maiores danos na cultura sem tratamento. Houve uma variação análoga nos IAF de plantas das parcelas tratadas e das parcelas não tratadas; sendo que aos 56 e 98 dias após o plantio tiveram-se os maiores índices de área foliar. A variação do IAF das plantas, em ambos os tratamentos, obedeceu uma curva semelhante à normalmente encontrada para a maioria das culturas (figura 1).

A relação de área foliar foi superior no tratamento com defensivo, sendo que esta relação mostra a eficiência do desenvolvimento da área foliar (figura 2). Deste modo verificou-se uma menor eficiência no desenvolvimento foliar no tratamento onde o nível de população de pragas foi mantido mais alto. Esta menor RAF ocorreu principalmente devido à intensa infestação de tripes na cultura; verificou-se que o aumento no nível de população deste inseto está relacionado com o incremento de folíolos novos.

No que se refere à taxa de assimilação aparente, observou-se que este parâmetro revelou valores superiores no tratamento sem defensivo. $O$ valor máximo da TAA foi obtido 42 dias após o plantio. Isto revela que o ganho em produtos de fotossíntese por unidade de área folia: e por unidade de tempo, é maior no tratamento sem defensivo (figura 3).

A taxa de crescimento relativo mostrou valores mais altos no tratamento sem inseticida, indicando que no tratamento sem defensivo a pro- 
dução de matéria seca por unidade foliar foi mais elevada que na região tratada (figura 4).

A variação da TAA e da TCR assumiu valores superiores no tratamento sem defensivo provavelmente porque o ataque das pragas torna as hastes das plantas mais rígidas, podendo aumentar consequentemente 0 peso seco das mesmas.

Verificou-se ainda um aumento de $15,8 \%$ na produção da região tratada, com relação a região onde não aplicou-se defensivo.

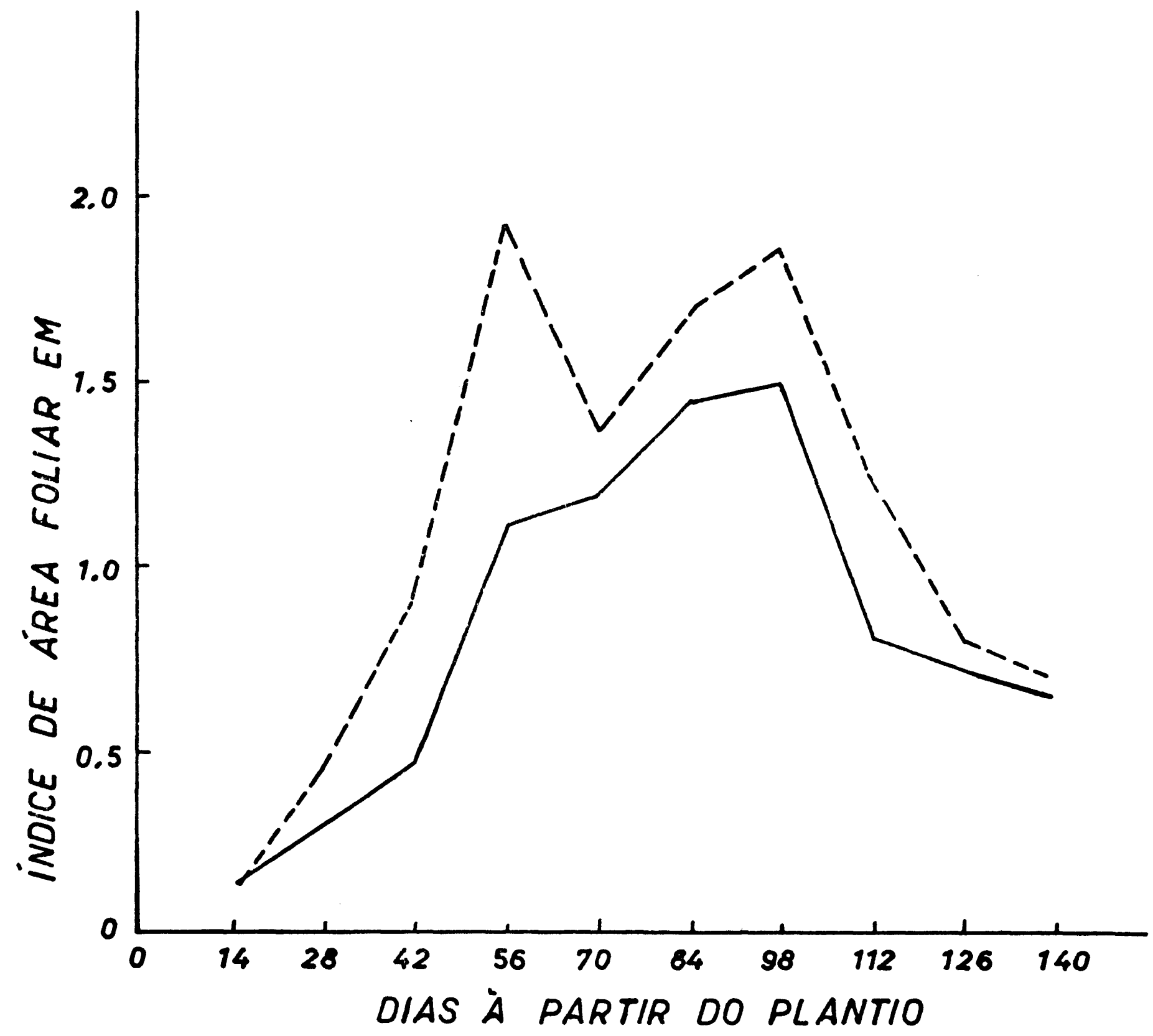

FIGURA 1. Indice de área foliar na área sem defensivo (-) e na área com defensivo (---), no decorrer do ciclo do amendoinzeiro. 


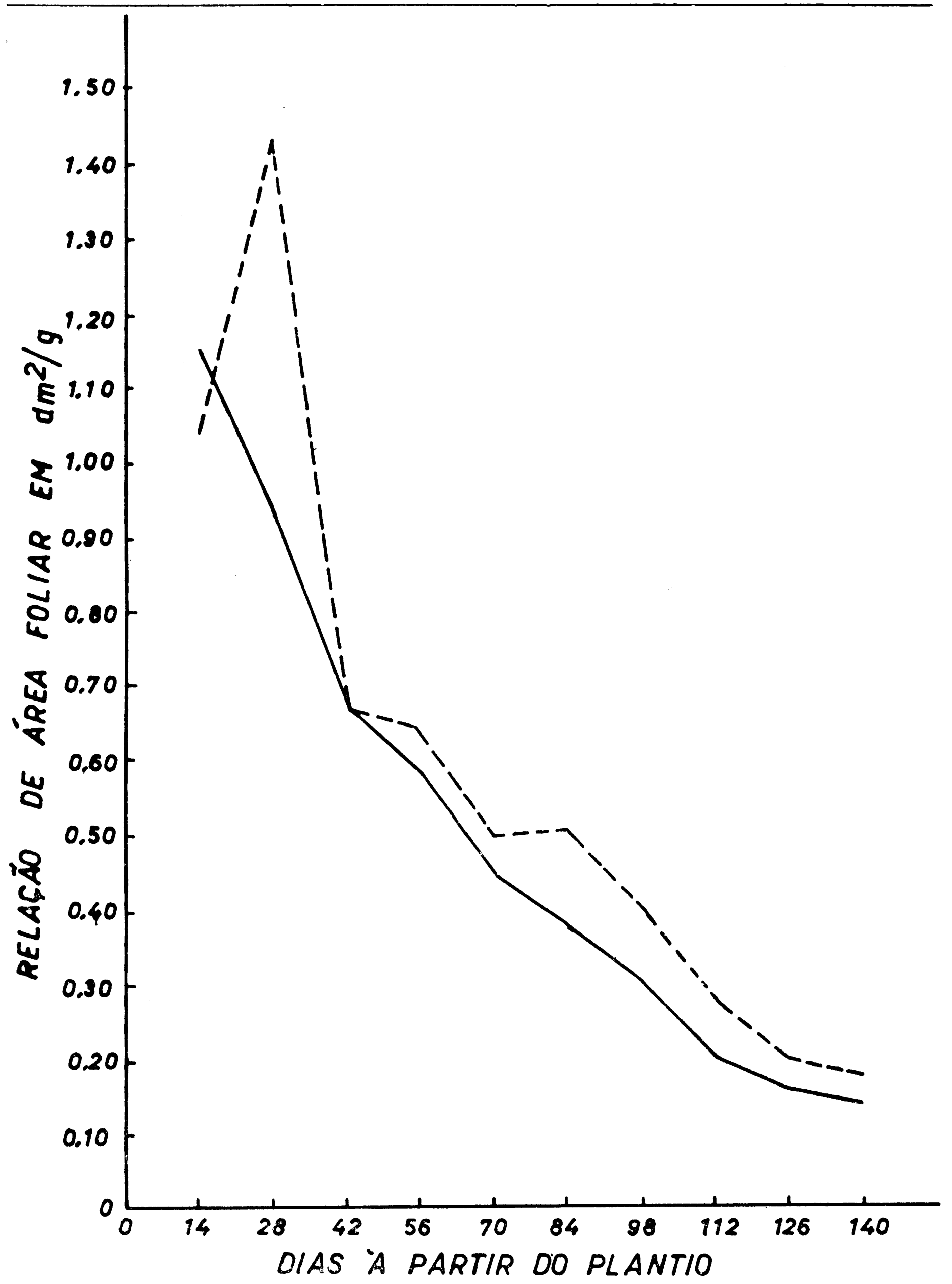

FIGURA 2. Relação de área foliar $\mathrm{em} \mathrm{dm}^{2} / \mathrm{g}$, na área sem defensivo (e na área com defensivo (-..-), no decorrer do ciclo do amendoinzeiro. 


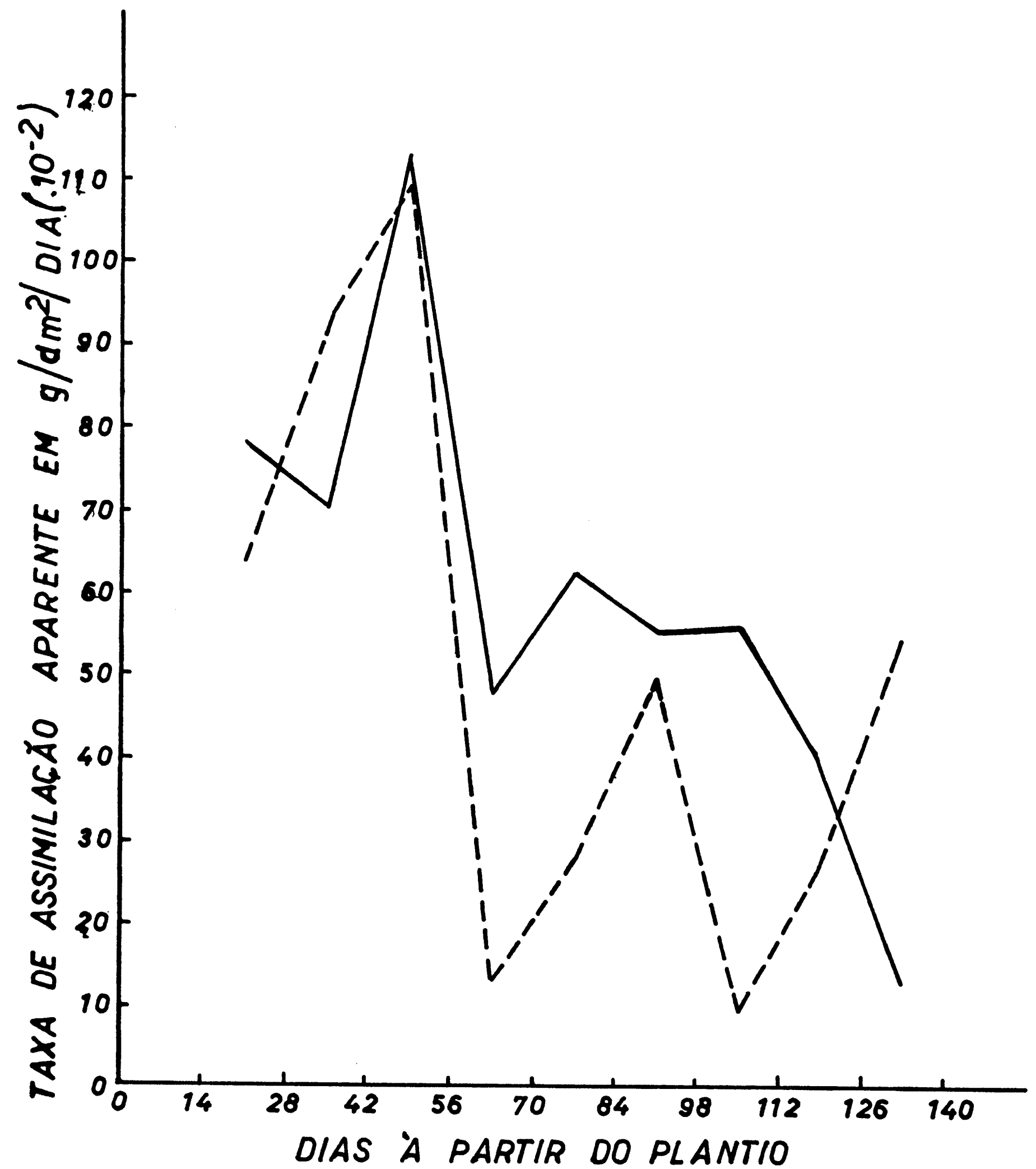

FIGURA 3. Taxa de assimilação aparente em $\mathrm{g} / \mathrm{dm}^{2} /$ dia $\left(.10^{-2}\right)$ na área sem defensivo ( $(-)$ e na área com defensivo (--), no decorrer do ciclo do amendoinzeiro. 


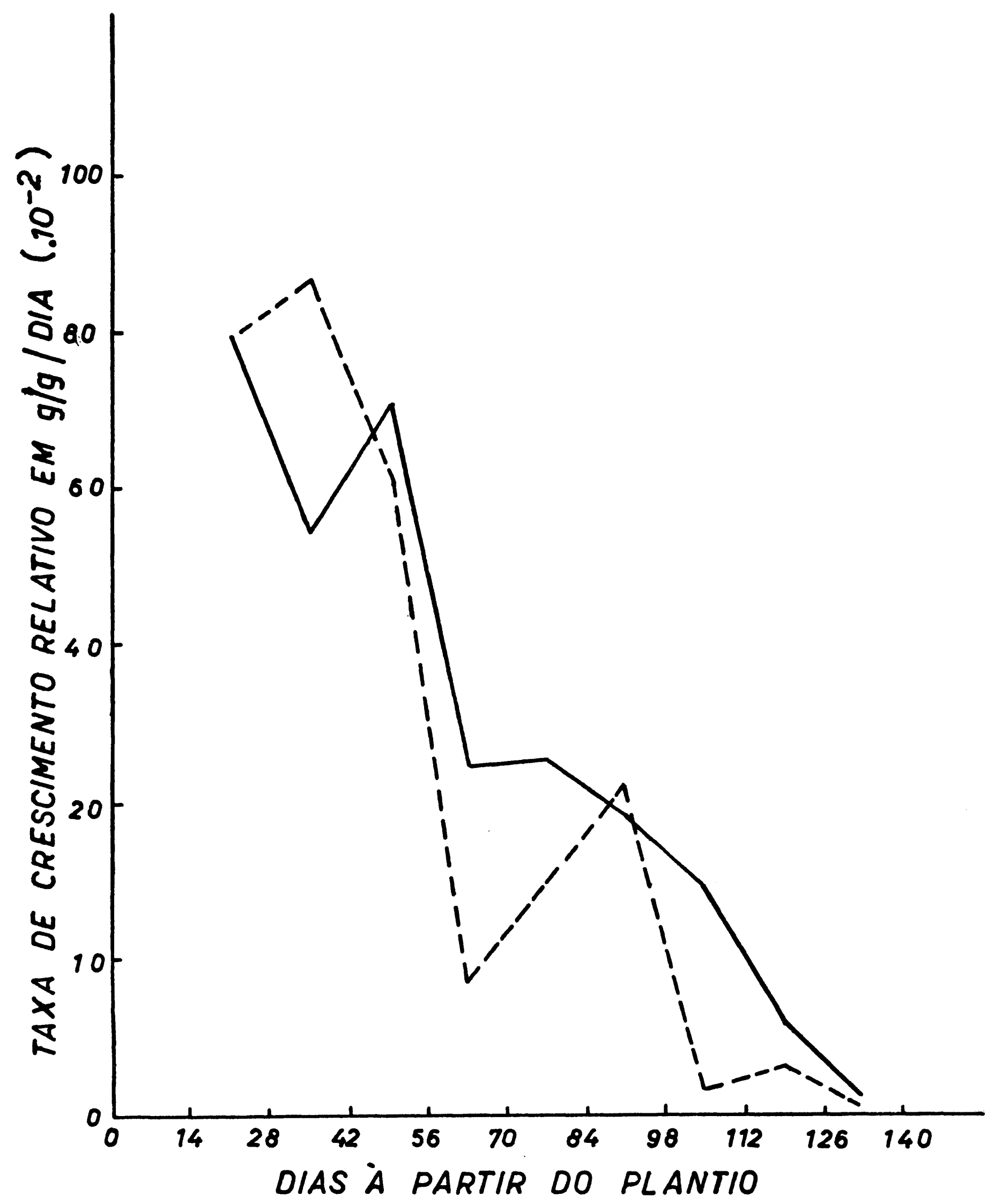

FIGURA 4. Taxa de crescimento relativo em $\mathrm{g} / \mathrm{g} / \mathrm{dia}\left(.10^{-2}\right)$ na área sem defensivo (-) e na área com defensivo (- --$)$ no decorrer do ciclo do amendoinzeiro. 


\section{CONCLUSOES}

Os resultados obtidos neste ensaio, permitem as seguintes conclusões:

1. Os danos provocados por pragas do amendoinzeiro diminuem o índice de área foliar da cultura.

2. Populações mais altas de pragas promovem decréscimo na relação de área foliar na cultura do amendoinzeiro.

3. A taxa de assimilação aparente e a taxa de crescimento relativo permanecem ligeiramente mais elevadas em amendoinzeiro sem defensivo.

\section{SUMMARY}

\section{GROWTH ANALYSIS OF PEANUT (Arachis hypogaea L.) WITH RELA- TION OF PESTS INFESTATION}

The peanut culture is one of the most important of our country. However it is studied with very little intensity. The thrips Enneothrips flavens Moulton, 1941, the leaf beetle Diabrotica speciosa (Germar, 1824) and the red necked peanut worm Stegasta bosquella (Chambers, 1875), are insect pests often found in peanuts (Arachis hypogaea L.), in Jaboticabal, State of São Paulo, Brazil.

The main proposal of this work is to study the variation of leaf area index, leaf area ratio, net assimilation rate and relative growth rate in an experiment in which one of the treatments consisted in the application of methyl parathion at $0,09 \%$ (E.C. $60 \%$ ) and the other was a check in which no pesticide was applied.

The results obtained during the plant life cycle were confronted. The increments of leaf area index and leaf area ratio were observed to be similar for both treatments, but both parameters were found to be practically always smaller for the check treatment.

Finally, it was shown that the net assimilation rate and the relative growth rate was almost always slightly superior in the plot that received no treatment.

\section{LITERATURA CITADA}

ALVIM, R. \& P. T. ALVIM 1969 - Efeito da densidade de plantio no aproveitamento da energia luminosa pelo milho (Zea mays) e pelo feijão (Phaseolus vulgaris), em culturas exclusivas e consorciadas. Turrialba $19(3): 389-393$.

BLACKMAN, G. E. \& G. L. WILSON 1951 - Physiological studies in the analysis of plant environment. VII. An analysis of the differential effects of light intensity on the net assimilation rate, leaf area ratio and relative growth rate of different species. Annals of Botany 15 (59) : 373-408.

BRANDES, D., M. MAESTRI, C. VIEIRA \& F. R. GOMES 1973 - Efeitos da po- 
pulação de plantas e da época de plantio no crescimento do feijoeiro (Phaseolus vulgaris L.) II. Anásise de crescimento. Experientiae 15 (1) : 1-30.

CASTRO, P. R. C., R. A. PITELLI \& R. L. PASSILONGO 1972 - Variações na ocorrência de algumas pragas do amendoinzeiro relacionadas com o desenvolvimento da cultura. Anais da Sociedade Entomológica do Brasil, Itabuna, BA, $1: 5-16$.

GRANGIER JR., A. \& P. T. ALVIM 1964 - Análise do crescimento e do vigor híbrido em plântulas de cacau Catongo. XV Congresso da Sociedade Botânica do Brasil, Porto Alegre, RS, 427-437.

HUERTA, A. S. \& P. T. ALVIM 1962 - Indice de área foliar y su influencia en la capacidad fotosintetica del cafeto. Cenicafé 13 (2) : 75-84.

MARSHALL, G. E., N. F. CHILDERS \& H. W. BRODY 1942 - The effects of leafhopper feeding injury on apparent photosynthesis and transpiration of apple leaves. Journal of Agricultural Research 65 (6) : 265-281.

MONSELISE, S. P. 1953 - Growth analysis of citrus seedlings. II. A comparison between sweet lime, rough lemon and sour orange. Palestine Journal of Botany $8: 125-132$.

MONTOJOS, J. C. \& A. C. MAGALhAES 1971 - Growth analysis of dry beans (Phaseolus vulgaris L. var. Pintado) under varying conditions of solar radiation and nitrogen application. Plant. and Soil $35: 217-223$.

VINOGRADOV, V. N. 1968 - Optimal leaf area of annual leguminous plants ensuring high crop yields. Fiziologia Rastenii $15(2): 361-362$.

WATSON, D. J. 1947 - Comparative physiological studies on the growth of field crops. I. Variation in net assimilation rate and leaf area between species and varieties, and within and between years. Annals of Botany $11: 41-76$. 
